\title{
Alternativa Para SimplificaÇão na Estrutura de ExeCuÇão de Projetos Seis-Sigma
}

\author{
Cristiano Marques de Oliveira ${ }^{1}$ \\ ${ }^{1}$ Delphi Automotive Systems \\ E-mails: cristiano.m.oliveira@ delphi.com
}

\section{RESUMO}

A rapidez na solução de problemas e melhorias de produtos e processos tem sido fundamental para garantir a competitividade no mercado atual. Dessa forma, a metodologia Seis-Sigma tem se mostrado como uma ferramenta poderosa para atingir esses objetivos com alta confiança.

Esse trabalho tem a intenção de apresentar uma alternativa aplicada para a simplificação na estrutura de execução de projetos Seis-Sigma, que visa facilitar a utilização da metodologia através do aumento da flexibilidade na sua estrutura sequencial.

\section{INTRODUÇÃO}

A capacidade de solucionar problemas com rapidez e qualidade se mostra um diferencial importante para o aumento da competitividade. Assim como em diversos setores da indústria, essa capacidade é fundamental no setor de autopeças, tendo em vista a grande concorrência atualmente presente no mercado.

É fundamental aumentar a competitividade de curto prazo no setor, sob risco de uma presença cada vez maior de autopeças importadas sendo introduzidas nos veículos fabricados no Brasil [1].

A competitividade refere-se à habilidade da empresa concorrer no mercado, à sua capacidade de igualar ou superar seus concorrentes na preferência dos consumidores. O potencial competitivo de uma economia pode ser avaliado a partir do exame dos fatores que condicionam a capacidade de suas empresas para o manejo eficaz desses mecanismos de competição [2].

Dentre desses fatores, pode-se destacar o nível de qualidade da educação como fortemente relacionado à habilidade analítica necessária na aplicação de metodologias de solução de problemas.

A metodologia Seis-Sigma é uma ferramenta poderosa para solução de problemas com alta confiança. A sua aplicação requer capacitação com relação à sua estrutura sequencial e principais ferramentas. 
Para facilitar a utilização da metodologia, uma alternativa de simplificação da sua estrutura de execução é proposta nesse artigo, com o intuito de remover barreiras e aumentar a frequência da sua aplicação.

\section{METODOLOGIA SEIS-SIGMA}

O conceito Seis-Sigma foi iniciado pela Motorola por volta de 1980, onde foram desenvolvidos métodos de solução de problemas que combinavam técnicas formais, particularmente relacionadas com medições, para atingir economias mensuráveis [3].

O nome Seis-Sigma deriva dos estudos de capabilidade e do desvio padrão do processo (sigma $\sigma$ ). A Figura 1 exemplifica um processo que apresenta seis desvios-padrão (seis sigma) entre a sua média e o limite de especificação mais próximo, dessa forma terá probabilidade próxima de zero de apresentar falhas.

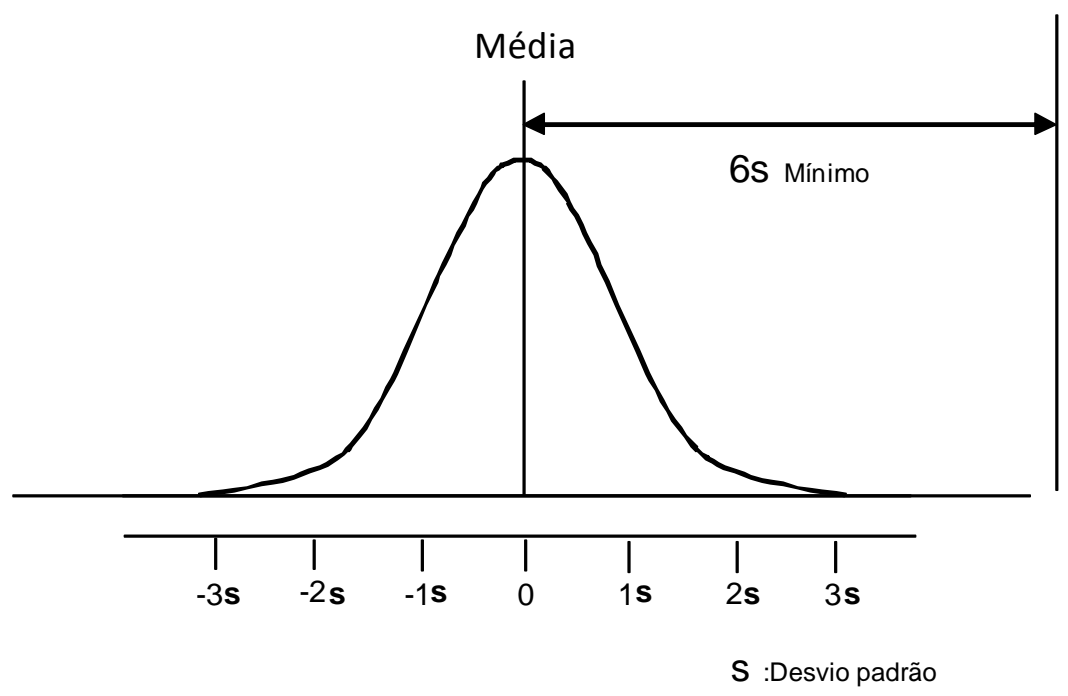

Figura 1: Processo Seis-Sigma

Além de uma metodologia para melhoria e solução de problemas, baseada na análise dos processos para encontrar as fontes de variação, o conceito Seis-Sigma também pode ser definido como um medidor e como um sistema de gestão. De acordo com a Figura 2, como um medidor é utilizado para medir o desempenho de um processo, como metodologia é uma forma estruturada de solução de problemas, já como sistema de gestão é focado na estratégia e melhoria contínua do negócio [4]. 


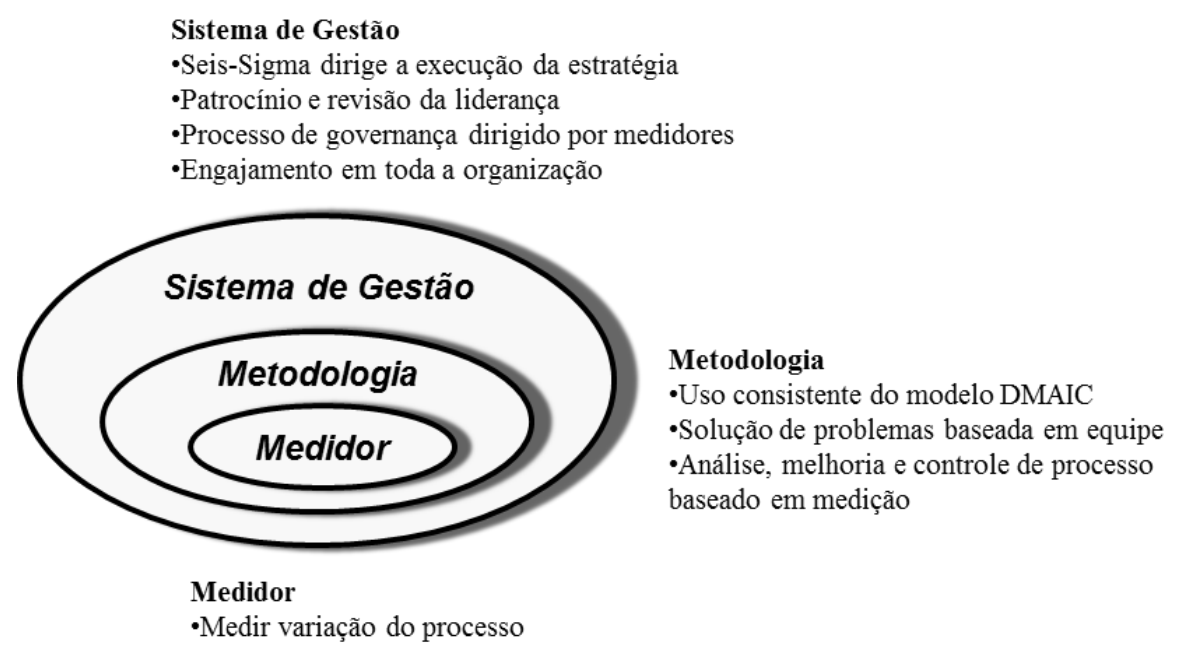

Figura 2: Seis-Sigma como Medidor, Metodologia e Sistema de Gestão [4]

Esse artigo se concentra na metodologia Seis-Sigma, principalmente na sua estrutura de execução.

\section{ESTRUTURA DMAIC}

A metodologia Seis-Sigma é estruturada em cinco fases com objetivos específicos. Essas fases são Definir (Define), Medir (Measure), Analisar (Analyze), Melhorar (Improve) e Controlar (Control). Essa estrutura é chamada de DMAIC.

A estrutura DMAIC, mostrada na Figura 3, fornece uma estrutura útil para a criação de processo com controle de passagem de fase. De acordo com os critérios para conclusão de cada fase em particular, revisões de projeto podem ser realizadas para verificar se os critérios foram atendidos antes de iniciar a próxima fase [5].

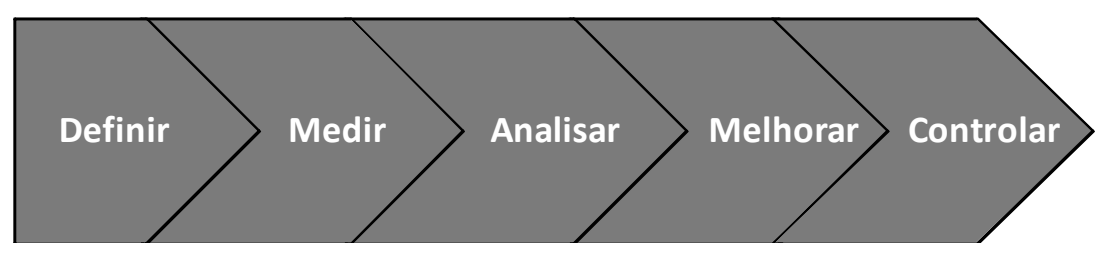

Figura 3: Ciclo de melhoria DMAIC

Cada fase tem seus objetivos específicos e ferramentas normalmente aplicadas. O conteúdo das fases fornece um método comum e estruturado para a solução de problemas. Para cada fase existem algumas atividades primárias e uma questão geral associada a ser respondida [4].

\subsection{DEFINIR (DEFINE)}

Nessa etapa os principais objetivos são definidos de forma preliminar, o contrato do projeto é assinado junto à liderança para obter o apoio necessário. Deve-se tomar cuidado com o escopo do projeto.

Questão importante: O que é importante para o negócio? [4] 
Principais ferramentas: Contrato, Diagrama de Afinidade, Matriz É / Não É, Mapa do Processo.

\subsection{MEDIR (MEASURE)}

Na fase de medição deve-se definir o plano de coleta de dados, assim como validar o sistema de medição a ser utilizado. A situação de desempenho atual deve ser mapeada de forma clara.

Questão: Como está indo com o processo atual? [4]

Principais ferramentas: Gráfico de Pareto, Histograma, Teste de Normalidade, Carta de Controle, Análise do Sistema de Medição, Estudo de Capabilidade.

\subsection{ANALISAR (ANALYZE)}

Múltiplas ferramentas são utilizadas com o intuito de encontrar e confirmar a causaraiz, com a melhor confiança possível. O ciclo apresentado na Figura 4 é executado e repetido até que se esteja confortável com a causa selecionada.

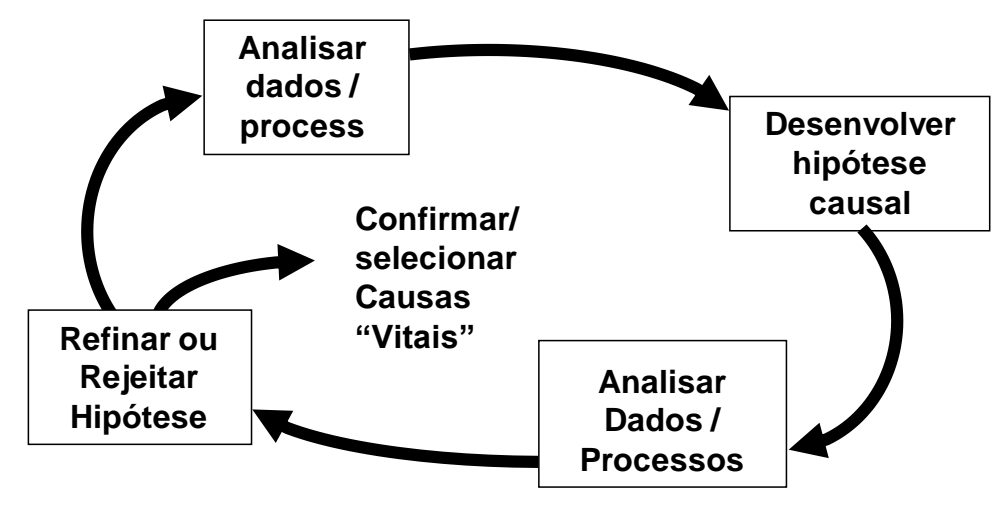

Figura 4: Ciclo de Análise da Causa Raiz

Questão: O que está de errado com o processo atual? [4]

Principais ferramentas: 5 Porquês, Diagrama de Causa e Efeito, Teste de Hipótese, Projeto de Experimentos, Regressão.

\subsection{MELHORAR (IMPROVE)}

São desenvolvidas e testadas soluções direcionadas para eliminar ou minimizar os efeitos relativos à causa selecionada na fase Analisar. Se necessário, são realizados testes piloto antes de mudanças definitivas nos processos ou produtos.

Questão: O que precisa ser feito para melhorar o processo? [4]

Principais ferramentas: Análise Custo/Benefício, Matriz de Seleção de Soluções, Projeto de Experimentos. 


\subsection{CONTROLAR (CONTROL)}

As melhorias se tornam parte do produto ou processo e são incorporadas na documentação oficial.

Questão: Como garantir que o desempenho das melhorias seja sustentável? [4]

Principais ferramentas: Plano de Controle, Instruções de Trabalho, Planilha Financeira, Estudo de Capabilidade.

\section{ESTRUTURA ALTERNATIVA}

Apesar de cada fase do ciclo DMAIC tem objetivos específicos, existe interação entre elas, o que torna necessária uma sistemática de retroalimentação conforme o andamento do projeto. Pela prática e experiência durante a execução dos projetos, observa-se que as três primeiras fases Definir, Medir e Analisar, podem ser entendidas como uma grande etapa com foco em encontrar e confirmar uma ou mais potenciais causas-raízes para o problema estudado.

Com o intuito de aumentar a flexibilidade sequencial, a estrutura alternativa proposta nesse artigo sugere o agrupamento das fases iniciais, Definir, Medir e Analisar em uma única fase de busca e confirmação da causa-raiz, como mostrado na Figura 5. Dessa forma, o levantamento de hipóteses e até mesmo possíveis soluções nas etapas iniciais do projeto passam a ser recomendas, diferente da estrutura tradicional DMAIC, onde essas ações não fazem parte das fases Definir e Medir.

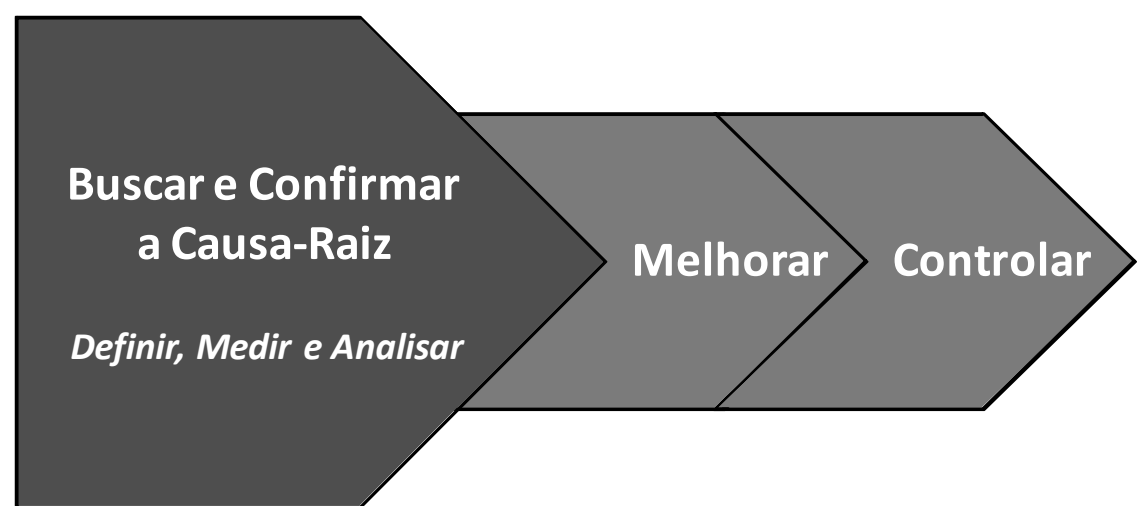

Figura 5: Estrutura Alternativa ao modelo DMAIC

A fase inicial de busca e confirmação da causa raiz permite a execução de mais atividades em paralelo com retroalimentação imediata. Na estrutura tradicional essas atividades seriam realizadas em série, como seria recomendado aguardar o final de uma fase para iniciar atividades da próxima etapa, essa condição poderia representar falta de agilidade relacionada à metodologia Seis-Sigma, prejudicando a sua utilização com maior frequência.

Um dos elementos motivadores para realizar o agrupamento das fases iniciais é incentivar o aumento do foco na buscar e confirmação da causa-raiz desde as primeiras ações do projeto. A avaliação de possíveis soluções já no início do ciclo também é amplamente incentivada. 


\subsection{NOVA FASE INICIAL}

A nova fase inicial apresentada na estrutura alternativa é baseada no ciclo de análise de causa raiz mostrado na Figura 4, onde se tem grande prioridade no desenvolvimento da hipótese de causa-raiz. Dessa forma não há restrição para que dados iniciais e potenciais soluções possam ser consideradas para o desenvolvimento de hipóteses preliminares.

As ferramentas utilizadas nessa etapa são as mesmas relacionadas anteriormente às fases Definir, Medir e Analisar da estrutura DMAIC, com a diferença da maior flexibilidade sequencial. Elas podem ser utilizadas paralelamente com o intuito de reunir dados do processo atual, suportar o desenvolvimento de hipóteses, garantir a validade dos dados analisados e confirmar com a confiança adequada uma possível causa-raiz vital a ser atacada.

Potenciais soluções identificadas através de experiências anteriores também podem consideradas no início do processo, sendo transformadas em hipóteses causais a serem avaliadas. Caso seja confirmada como uma causa-raiz vital, a etapa de melhoria pode ser iniciada com grande agilidade.

A Figura 6 mostra o fluxo de execução de projeto conforme a estrutura alternativa, considerando a nova fase inicial e as suas principais atividades.

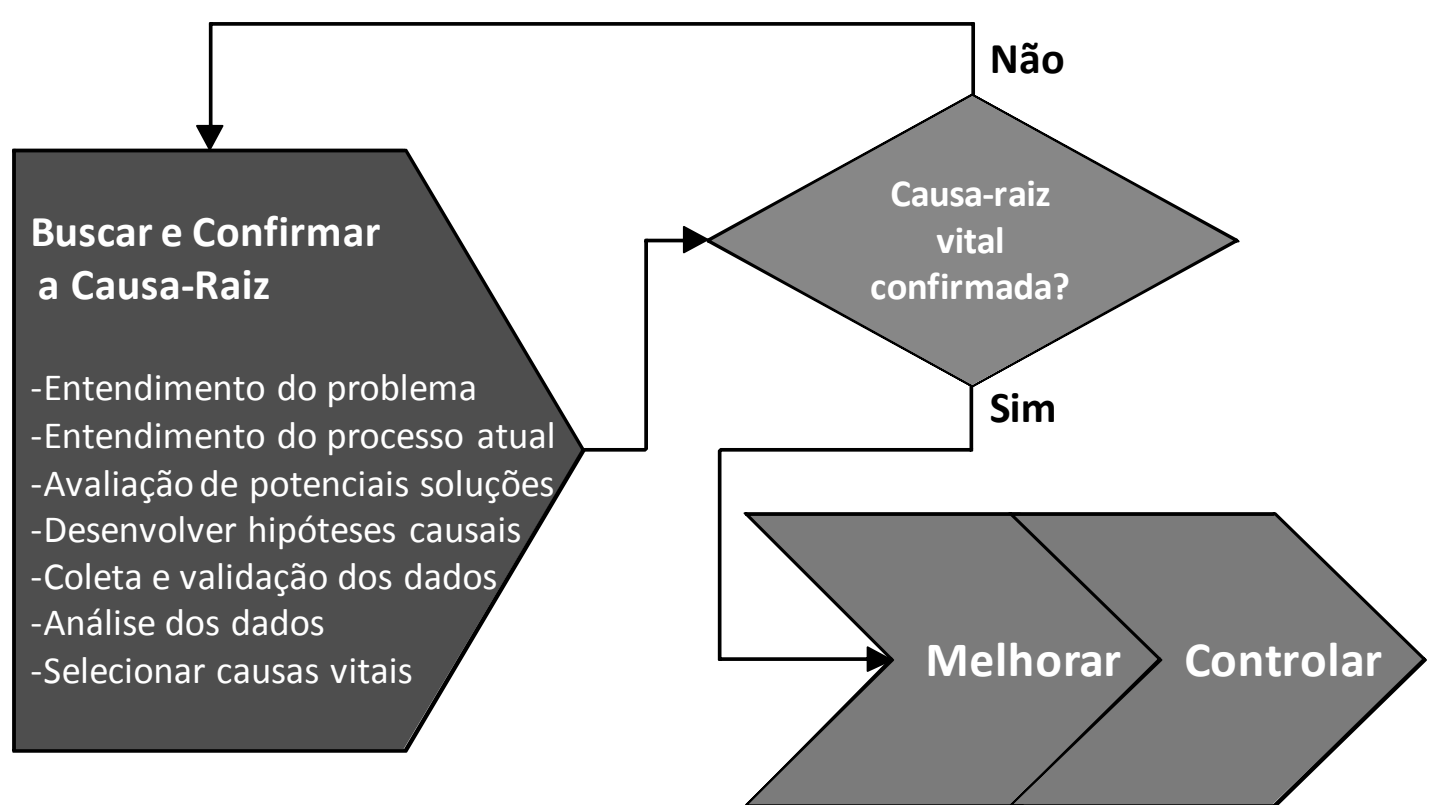

Figura 6: Fluxo de Execução de Projeto

Como mencionado anteriormente, para cada fase da estrutura DMAIC existe uma questão geral associada a ser respondida. Logo para a nova fase apresentada deve ser associada uma nova questão, considerando a sua flexibilidade sequencial.

Nova questão a ser respondida: Por que o desempenho observado do processo atual não está de acordo com a necessidade do negócio? 


\subsection{COMPARATIVO}

Além do agrupamento das fases, pode-se notar que o foco inicial da estrutura alternativa também apresenta diferença com relação ao DMAIC. Na estrutura DMAIC o foco inicial está na definição do escopo, enquanto na estrutura alternativa, desde o início o foco é a seleção de uma causa-vital, para poder iniciar a fase Melhorar o mais rápido possível.

A Tabela 1 mostra de forma resumida o comparativo entre o foco das fases Definir, Medir e Analisar da estrutura DMAIC e o foco da fase inicial Buscar e Confirmar a Causa-Raiz da estrutura alternativa.

\begin{tabular}{|l|l|}
\hline Estrutura DMAIC & Estrutura Alternativa \\
\hline $\begin{array}{l}\text { Fase Definir: foco gerencial na } \\
\text { definição do problema, planejamento e e } \\
\text { escopo do projeto. }\end{array}$ & $\begin{array}{l}\text { Fase Buscar \& Confirmar: foco em } \\
\text { testar hipóteses para encontrar a causa- } \\
\text { raiz vital. Utilização de ferramentas de } \\
\text { definição, medição e análise em } \\
\text { paralelo conforme necessidade do } \\
\text { projeto. Potenciais soluções são bem- } \\
\text { vindas nessa etapa, pois podem ser } \\
\text { sistema de medição, coleta de dados } \\
\text { referentes à situação atual. }\end{array}$ \\
$\begin{array}{l}\text { Fase Analisar: iniciada a investigação } \\
\text { tas potenciais causas para busca da } \\
\text { causa-raiz vital. }\end{array}$ & $\begin{array}{l}\text { sermadas em hipóteses causais e } \\
\text { serem testadas. }\end{array}$ \\
\hline
\end{tabular}

Tabela 1: Comparativo entre as fases Definir, Medir e Analisar da estrutura DMAIC e a nova fase inicial Buscar \& Confirmar causa-raiz da estrutura alternativa proposta.

A estrutura alternativa se mostra bastante adequada para solução de problemas durante o desenvolvimento de produtos e processos dentro da área de Engenharia, uma vez os responsáveis técnicos poderão estar diretamente envolvidos na solução do problema em questão.

As informações geradas durante o processo de desenvolvimento podem fornecer os dados iniciais bastante completos, incluindo resultados de medições, para que seja possível ter o foco na busca pela causa-raiz desde o momento inicial quando identificada a necessidade do projeto Seis-Sigma.

\section{RESULTADOS E PERCEPÇÕES INICIAIS}

Como resultado do uso da estrutura alternativa, houve aumento na maior quantidade de projetos Seis-Sigma, uma vez que a abordagem intuitiva de solução de problema, com a identificação de causas potenciais no início do processo, pode ser convertida mais facilmente na metodologia Seis-Sigma sem criação de barreiras.

Com a nova estrutura, potenciais soluções levantadas nas fases iniciais podem ser reformuladas como causa potencial e, dessa forma, podem ser testadas já no início do projeto. Essa maior flexibilidade na estrutura beneficiou a aceitação e a busca pelo uso da metodologia, mantendo ganho de confiança nos resultados com maior agilidade. 


\section{CONCLUSÃO}

A estrutura alternativa tem sido utilizada dentro da área de Engenharia de forma controlada com resultados positivos, porém ainda é necessária maior utilização para confirmação da sua efetividade em maior escala. A simplificação da estrutura de execução foi efetiva e sem prejuízo nos objetivos alcançados nos projetos.

Devido às atividades em paralelo, é necessário que o executor certificado na metodologia Seis-Sigma tenha claramente o objetivo a ser atingido e as ferramentas necessárias de forma interativa.

Com a utilização da estrutura alternativa e com foco aumentado efetivamente no mapeamento da causa e na solução do problema, a percepção de agilidade relativa ao uso da metodologia Seis-Sigma pode ser beneficiada.

Existe a oportunidade de aplicação do novo conceito também para a área de inovação, dentro da metodologia Projeto para Seis-Sigma (Design For Six-Sigma), utilizada para a criação de produtos e processo já com o critério Seis-Sigma.

Para esclarecimentos adicionais, contatar:

Cristiano Marques de Oliveira

Delphi Automotive Systems

Phone: +55 (12) 3978-2006

E-Mail: cristiano.m.oliveira@delphi.com

\section{REFERÊNCIAS}

[1] ABDI - Agência Brasileira de Desenvolvimento Industrial, "Estudo Prospectivo Setorial Automotivo," Brasília, 2009.

[2] CNI - Confederação Nacional da Indústria, "Competitividade Brasil 2013," Brasília, 2013.

[3] ALLEN, Theodore T., "Introduction to engineering statistics and six sigma: statistical quality control and design of experiments and systems," Springer-Verlag London, 2006, ISBN-10: 1852339551.

[4] McCARTY, T., Bremer, M. Daniels, L. and Gupta, P., "The Six Sigma Black Belt Handbook," McGraw-Hill, 2004.

[5] PYZDEK, Thomas and Keller, Paul, "The Six Sigma Handbook," McGraw-Hill, 3rd edition, 2010. 